\title{
Individual loudness functions determined from direct comparisons of loudness intervals
}

\author{
BRUCE SCHNEIDER \\ University of Toronto, Mississauga, Ontario, Canada LSL IC6
}

\begin{abstract}
Five subjects were required in each trial to directly compare two pairs of tones and indicate which pair of tones had the greater loudness difference. Ten $1,200-\mathrm{Hz}$ tones differing only in intensity were employed. Subjects made binary comparisons among the 45 tone pairs that can be formed from these 10 tones. The loudness difference comparisons of each subject were found to satisfy two properties (transitivity and monotonicity) that are required for an interval scale representation of loudness. Therefore, individual loudness scales were constructed using a nonmetric scaling technique designed for comparisons of sensory intervals. These loudness scales differed significantly from subject to subject. Since a nonnumerical scaling procedure was employed, these individual differences could not be attributed to biases in the way in which observers use numbers or numerical concepts to describe the loudness of tones. Hence, they suggest strong individual differences in the coding of sound intensity.
\end{abstract}

Most investigations concerned with the measurement of loudness have employed the direct estimation methods advocated by S. S. Stevens $(1955,1956)$. These methods require that the observer, in one fashion or another, assign a number to represent the loudness of a sound. For example, in a magnitude estimation task, observers are asked to assign numbers to stimuli such that the ratio of any two numbers is the ratio of the loudnesses of the corresponding sounds. Numerical judgments obtained in this fashion are found to be a power function of sound intensity, that is,

$$
\mathrm{N}=k \mathrm{I}^{\mathrm{n}}
$$

where $\mathbf{N}$ represents the observer's numerical judgment, I denotes sound intensity, and $k$ and $n$ are constants. The sone scale of loudness is to a large extent a product of these methods.

However, in order to argue that direct scaling techniques such as magnitude estimation actually measure sensory intensity, it is necessary to assume that the numerical judgments are directly proportional to loudness. Several investigators have argued that this assumption is unwarranted, that is, that numerical estimates are biased in one fashion or another. For example, Marks (1974), in reviewing 27 studies involving direct scaling of loudness, noted that the value of the exponent (as a function of sound intensity)

This research was supported by a grant from the Natural Sciences and Engineering Research Council of Canada. Reprints can be obtained by writing to B. Schneider, Department of Psychology, Erindale College, University of Toronto, Mississauga, Ontario L.5L IC6, Canada. ranged from .185 to .425 , a range of over 2 to 1 . If we assume that the sensory system does not change from procedure to procedure or condition to condition, we are left with the conclusion that subjects may not always apply numbers or numerical concepts in a linear fashion. In addition, several experiments involving judgments of sensory differences, sensory ratios, and sensory sums have also pointed to the existence of response biases in numerical estimates (Anderson, 1974; Curtis, Attneave, \& Harrington, 1968; Curtis \& Rule, 1972; Fagot, Stewart, \& Kleinknecht, 1975; Rule, Curtis, \& Markley, 1970; Rule, Laye, \& Curtis, 1974; Schneider, Parker, Valenti, Farrell, \& Kanow, 1978).

If there are indeed biases in the way in which subjects apply numbers or numerical concepts, these biases must be taken into account in our attempts to measure loudness. S. S. Stevens (e.g., 1971) has argued that a balanced average of a large number of experiments using direct scaling procedures can yield valid measurement scales. The logic of this argument is that although any single procedure may be biased, the average of a few carefully chosen procedures will produce an unbiased result. A second approach is to model the kind of response bias that occurs so that it can be evaluated and removed (e.g., Fagot, Stewart, \& Kleinknecht, 1975; Rule, Laye, \& Curtis, 1974). A third approach, and the one that is employed here, is to use a procedure that dispenses with numerical judgments and thereby circumvents the problem of numerical biases.

\section{Paired Comparisons of Sensory Intervals}

The use of numbers or numerical concepts in psychophysical judgments can be avoided by using a 
paired comparison procedure. For example, one could simply require an observer to compare two stimuli and indicate which one is louder. This requires only a binary judgment on the part of the subject and does not require the use of numbers or numerical concepts. Hence, there is no need to worry about numerical biases "distorting" the loudness scale. Unfortunately, only an ordinal scale of loudness can be constructed in this fashion. Since the ordinal properties of the loudness of pure tones of fixed frequency already are specified by their intensity values, little would be gained by such an effort. Krantz, Luce, Suppes, and Tversky (1971), however, have shown that if loudness intervals of pairs of tones rather than the loudnesses of two tones are compared, it is possible to construct an interval scale of loudness. An example of an experiment of this sort is provided by Schneider, Parker, and Stein (1974). In this experiment, subjects were required, on each trial, to directly compare two pairs of tones and indicate which pair had the greater loudness difference. Ten $1,200-\mathrm{Hz}$ tones differing only in intensity were employed. Subjects made binary comparisons among the 45 tone pairs that were formed from the set of 10 tones. Hence, in each trial the subjects were required to judge whether the tones in pair A or pair B were more widely separated in loudness. Since only a binary comparison was required, numerical judgments and related numerical concepts were avoided.

In addition to avoiding the use of numbers, there are also two other advantages to this paired comparison design. First, it permits two tests as to whether the subject is actually judging loudness intervals in a fashion that is consistent with the instructions. Second, it provides a means of determining interval scale values for the loudnesses of the tones. These advantages allow the determination of an interval scale of loudness without resorting to the use of numbers or numerical concepts by the observer.

\section{Axiomatic Tests for Comparisons of Sensory Intervals}

If subjects are actually comparing loudness intervals, they should respond that pair $(x, y)$ has a loudness interval as great as or greater than some other pair $(w, z)$ if and only if the loudness difference, $D(x, y)$, between tones $\mathrm{x}$ and $\mathrm{y}$ is as great as or greater than the loudness difference, $D(w, z)$, between tones $w$ and $\mathrm{z}$, that is

$$
(x, y) \geqslant(w, z) \text { iff } D(x, y) \geqslant D(w, z) .
$$

In order to insure that loudness differences are always positive, we adopt the convention that the designation for a tonal pair whose elements are $\mathrm{x}$ and $y$ is $(x, y)$ if and only if the intensity of $x$ is strictly less than the intensity of $y$. This convention allows us to represent loudness differences as positive intervals or differences where $D(x, y)$ is defined as $L(y)$ $L(x)$, the algebraic difference in the loudnesses of the two tones. The convention is reasonable, given that the loudness of a pure tone of fixed frequency is monotonic with intensity.

Since loudness is unidimensional, we should be able to represent the loudnesses of tones as points on a line segment where the distance between points represents the experience of loudness difference. Therefore, comparisons of loudness intervals should behave as if they were comparisons of distances along a line segment. Krantz et al. (1971) have indicated two critical properties of distances that difference judgments must satisfy if interval scale measurement is to be achieved. The first is transitivity, that is,

$$
\begin{aligned}
(r, s) \geqslant(t, u) \text { and }(t, u) \geqslant & (x, y) \\
& \quad \text { implies }(r, s) \geqslant(x, y) .
\end{aligned}
$$

The second is the so-called monotonicity condition. This axiom states that for $a \leqslant b \leqslant c$ and $x \leqslant y \leqslant z$,

$$
\begin{aligned}
(a, b) \geqslant(x, y) \text { and }(b, c) \geqslant & (y, z) \\
& \text { implies }(a, c) \geqslant(x, z) .
\end{aligned}
$$

This condition is illustrated in Figure 1 and is fairly obvious geometrically. Krantz et al.(1971) have shown that these two axioms (or their equivalent) must be satisfied in order to permit interval scale measurement of the underlying sensory dimension. Schneider et al. (1974) showed that for group data, these two axioms were essentially satisfied, thus permitting an interval scale representation of loudness. However, since only one judgment per interval comparison was obtained from individual subjects, it was not possible to determine whether these two properties also held true for individual subjects. Consequently,
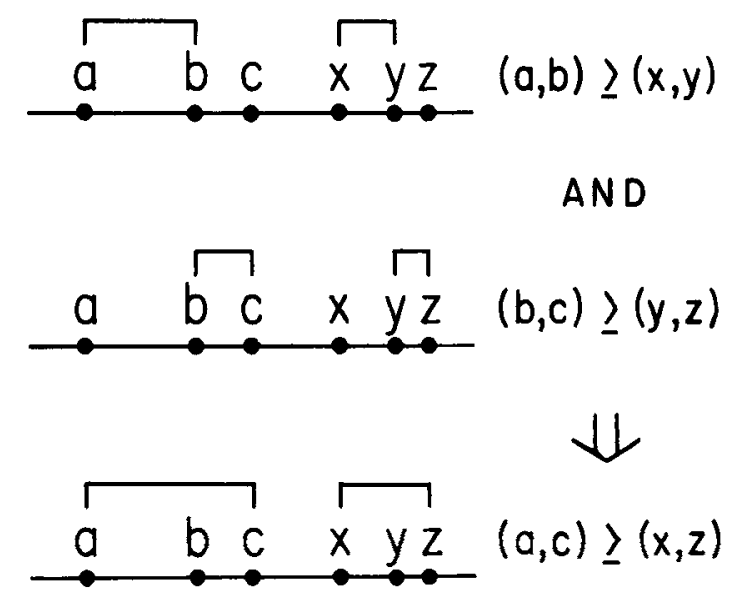

Figure 1. Illustration of the monolonicity condition for points on a line segment. 
one of the goals of the present experiment was to see whether individual subjects can judge sensory intervals in a way that is consistent with the instructions (satisfy these two conditions), thus permitting the construction of interval scales of loudness for these individuals.

\section{Deriving Loudness Scales from \\ Comparisons of Sensory Intervals}

Shepard (1966) has shown that, provided the number of stimuli is $\geqslant 10$, the rank order of a set of interpoint distances can be used to determine projection values along a line segment, which are, for all practical purposes, unique up to addition and multiplication by a constant. If loudness interval comparisons behave as distances and satisfy Properties 3 and 4 , then the comparisons can be used to generate a rank order of sensory intervals that can be interpreted as a rank order of distances along a line segment. Consequently, projection values along such a line segment obtained from this rank order would represent an interval scale of loudness. Schneider et al. (1974) found that the loudness scale thus obtained for the group data was linearly related to the .15 power of sound intensity. ${ }^{1}$

However, Schneider et al. (1974) were unable to apply this kind of analysis to the individual subjects because, with only one judgment per comparison, it proved impossible to determine a representative rank order of the loudness intervals for individual subjects. Since individual subject data also were not tested for transitivity and monotonicity, this study could not address any questions concerning individual differences in the ability to compare loudness intervals. In the present paper, 4,320 comparisons of loudness intervals were obtained from each of five subjects. These comparisons are used to determine whether the judgments of individual subjects satisfy transitivity and monotonicity, and whether there are any differences in the loudness scales across subjects. J. C. Stevens and Guirao (1964), using an estimationproduction combination, have shown that exponents obtained from individual subjects vary considerably. Even when direct numerical judgments are avoided, as in cross-modality matching experiments, individual differences still occur (Marks \& Stevens, 1966; Rule \& Markley, 1971; Walsh \& Browman, 1978; Wanschura \& Dawson, 1974) and sometimes are substantial. It would be interesting to determine whether the elimination of numerical judgments or concepts in the direct comparison of loudness intervals would reduce or eliminate large individual differences. If individual differences are minimized, it would suggest that individual differences in the loudness functions obtained from direct scaling procedures primarily reflect numerical biases. If, however, individual differences still appear, it would suggest that there are differences in the coding of sound in- tensity across individuals. The present study, then, will enable us to construct interval scales of loudness for individual subjects from binary comparisons of loudness intervals and to determine the extent to which individual differences are minimized through the use of a nonnumerical scaling technique.

\section{EXPERIMENT}

\section{Method}

Subjects. The author, two graduate students, and two research assistants served as subjects. All had previous experience in auditory psychophysical tasks.

Apparatus. A pure tone $(1,200 \mathrm{~Hz})$ was generated by a HewlettPackard oscillator (Model 204C). The output of the oscillator was gated by a Grason-Stadler electronic switch (Model 1287B) with a rise and decay time of $10 \mathrm{msec}$ to dampen transients. The output of the electronic switch, after passing through an amplifier (Tektronix, Model AM502) was gated in sequence through attenuators 1 and 2 , when the subject pressed button 1 . When this button was pressed, the output of the amplifier was connected to attenuator 1 , and the output of attenuator 1 was connected to the earphone. Fifty milliseconds later, the electronic switch was turned on for a period of $750 \mathrm{msec}$. As a result, a 750 -msec tone was presented to the subject, with the intensity determined by the dial setting of attenuator 1. Fifty milliseconds after the tone was turned off, the output of the amplifier was disconnected from attenuator 1 , and, at the same time, attenuator 1 was disconnected from the earphone. This sequence was repeated $800 \mathrm{msec}$ later, with the tone now being switched through attenuator 2. As far as the subject was concerned, pressing button 1 resulted in the presentation of two $750-\mathrm{msec}$ tones differing in intensity and separated by a period of $900 \mathrm{msec}$. There was a 300 -msec delay following the presentation of the second tone before presses on either button would be effective. If button 2 was pressed, the output of the electronic switch was gated through attenuators 3 and 4 , respectively, to produce two different tones. The subject was allowed to present himself with either pair of tones any number of times he wished in order to arrive at a judgment as to which pair had the greater loudness difference.

At the beginning of a trial, the experimenter set up the four intensities of the tones via attenuators 1-4. Signal frequency and voltage were monitored with a Tektronix counter (Model DC503) and voltmeter (Model DM501). Signal waveform was monitored on an oscilloscope. Voltage levels were converted to sound pressure via earphone calibrations performed by the National Bureau of Standards in Washington, D.C., with an NBS 9-A coupler. Listening was monaural (right ear) throughout the entire experiment. The subject sat inside an Industrial Acoustics singlewalled sound-attenuating chamber and communicated with the experimenter via an intercom.

Procedure. Subjects were requested to judge which of two pairs of tones had the greater absolute difference in loudness. The tonal frequency, tonal duration, and intertone time were identical to those of Schneider et al. (1974). Comparisons were made among tone pairs constructed from the following set of $1,200-\mathrm{Hz}$ tones: $49,55,59,67,71,79,85,93,97$, and $103 \mathrm{~dB}$. With 10 tones, there are 45 distinct pairs. If every such pair of tones is compared with every other pair, there are 990 comparisons among pairs of tones. However, following Schneider et al. (1974), only the 540 critical comparisons out of the 990 possible comparisons were presented to the subject. This reduction is made possible by the fact that the loudnesses of the $1,200-\mathrm{Hz}$ tones are monotonic with intensity. Hence, the tones can be ordered from loudest to softest. Consequenily, we can assume that any pair of tones whose intensities are bracketed on either side by another pair of tones will be judged to have a smaller loudness difference than the tones that bracket this pair. For example, 
consider a comparison between a 50-75-dB interval and an interval of $50-73 \mathrm{~dB}$. If the tones vary along a single psychological dimension, and if their loudnesses are monotonic with intensity, then the 50-75-dB interval will have a greater loudness difference than the 50-73-dB interval. Therefore, it was not necessary to compare pairs of tones when the intensity interval of one pair was "nested" within the intensity interval of another pair. Eliminating these "nested" comparisons reduced the number of comparisons to 540 . Each subject listened to each of these 540 comparisons a total of eight times over the course of 64 sessions.

During the first eight sessions, a subject was presented once with each of the 540 pairs of tones in a random order. The first seven test sessions contained 70 tone pairs and the eighth contained 50 . In each of the following seven replications of these eight sessions, the 540 tones were presented in a different random order. Since the four tones presented in each paired comparison could occur in eight different arrangements $(a, b: x, y ; b, a: x, y ; a, b: y, x ; b, a: y, x$; $x, y: a, b ; y, x: a, b ; x, y: b, a ; y, x: b, a)$, these eight arrangements for a comparison of tone pairs were assigned randomly across the eight replications. Hence, order effects were balanced across replications within an individual. Sessions generally lasted 45-80 min with a 10-min break halfway through the session.

\section{Results}

Transitivity and monotonicity. The data from each subject consisted of the number of times he or she judged one pair of tones to have a greater loudness difference than another for each of the 540 comparisons. If pair $(r, s)$ was judged to have a greater loudness interval than pair $(t, u)$ on more than one-half of the trials, then we write $(r, s)>(t, u)$. Occasionally, ties occurred; that is, $(r, s)$ was judged to have a greater interval than $(t, u)$ on exactly one-half of the trials. In that case, $(r, s)=(t, u)$. The number of such ties was small and is shown in Table 1 for each subject and for the group data. To check for transitivity, ranks were assigned to the tone pairs such that whenever

$$
\begin{aligned}
& (r, s) \geqslant(t, u) \text { and }(t, u) \geqslant(x, y) \\
& \text { then } N(r, s) \geqslant N(t, u) \geqslant N(x, y),
\end{aligned}
$$

where $\mathrm{N}$ stands for a numerical assignment. If there are no violations of transitivity, then the intervals can be assigned a numerical rank without any violations of Equation 5. If, on the other hand, there are violations of transitivity, there will be instances in which it is not possible to maintain this assignment procedure. The number of such instances indicates the extent to which transitivity is violated. Hence, for each subject and for the group data, rank orders were sought that resulted in the minimal number of violations of Equation 5. Table 1 (column 2) presents the number of violations for the five subjects and for the group data. ${ }^{2}$ The number of violations of transitivity ranged from one for Subject R.S. to seven for Subject J.F. Given that there are so few violations of transitivity for each subject and for the group data, we can conclude that the transitivity requirement is essentially satisfied.

To test the monotonicity requirement, we examined all possible cases in which violations of monotonicity could occur. For the number of comparisons involved here, there are 2,520 instances in all. Since not all of these instances actually provide tests of the monotonicity requirement, we counted for each subject the number of times when the monotonicity condition should hold (the number of cases in which the first half of Equation 4 was satisfied) and the number of times it failed to hold. Column 3 of Table 1 gives the total number of testable cases for monotonicity, while column 4 gives the percentage of failures of this condition. The failure rate ranges from $.3 \%$ to $2.2 \%$. Hence, we can conclude that judgments of loudness differences from individual subjects satisfy both weak transitivity and monotonicity.

Loudness scale values. A nonmetric program designed for paired comparisons of differences (Schneider, 1980) was used to determine scale values for the 10 stimuli for each of the five subjects and for the group data. This program arranges the stimuli on a line segment such that the distances between stimuli on the line segment best predict the paired comparisons data; that is, it arranges the stimuli along the line segment such that, for all pairs of intervals, whenever $(x, y)>(w, z)$ then $|P(y)-P(x)|>$ $|P(z)-P(w)|$, where $P(x)$ is the coordinate value of $\mathrm{x}$ along the line segment produced by the program. Of course, in errorful data it is not possible to do this perfectly; hence, the program minimizes the number of disagreements between the predicted paired comparisons and the obtained paired comparisons. Formally, the program minimizes the quantity, $G=$

\begin{tabular}{|c|c|c|c|c|c|c|c|}
\hline Subject & Ties & $\begin{array}{l}\text { Violations } \\
\text { of Tran- } \\
\text { sitivity }\end{array}$ & $\begin{array}{c}\text { Testable } \\
\text { Cases of } \\
\text { Monotonicity }\end{array}$ & $\begin{array}{l}\text { Percentage } \\
\text { Failure of } \\
\text { Monotonicity }\end{array}$ & G & $\begin{array}{l}\text { Bounds on } \\
\text { Estimate } \\
\text { of CM }\end{array}$ & Exponent \\
\hline E.D. & 32 & 6 & 1527 & 2.2 & .019 & $.996-.999$ & .11 \\
\hline J.F. & 29 & 7 & 1961 & 1.1 & .019 & $.997-.999$ & .24 \\
\hline D.K. & 38 & 3 & 1965 & 1.0 & .019 & $.995-.999$ & .28 \\
\hline B.S. & 18 & 2 & 2141 & .3 & .006 & $.998-.999$ & .26 \\
\hline R.S. & 12 & 1 & 1834 & 1.1 & .017 & $.998-.999$ & .20 \\
\hline Group & 5 & 2 & 1832 & .9 & .015 & $.999-.999$ & .21 \\
\hline
\end{tabular}

Table 1 
$N_{d} /\left(N_{r}-N_{t}\right)$, where $N_{d}$ is the number of disagreements, $N_{r}$ is the number of paired comparisons, and $N_{t}$ is the number of cases in which the predicted comparisons are indeterminate, that is, $|P(y)-P(x)|=$ $|P(z)-P(w)|$. Values of $G$ are shown in Table 1 .

These values of $G$ can be used to estimate the degree to which the projection values obtained from this program actually represent interval scale measurement. To accomplish this, the index of coordinate metric determinacy (CM) was estimated from Schneider's (1980) nomogram. CM is the squared Pearson correlation coefficient between the true coordinate values (which presumably generated the obtained paired comparisons) and the projection values produced by the program. Hence, $\mathrm{CM}$ varies between 0 and 1 , and $C M=1$ means that the true coordinate values have been perfectly recovered. In no empirical investigation using these techniques are the true coordinate values known, but Schneider (1980) provides a nomogram for estimating $\mathrm{CM}$ from the number of points, and the value of $\mathrm{G}$, both of which are available in an empirical study. Hence, if the estimated value of $\mathrm{CM}$ is sufficiently high, the point coordinates produced by the algorithm are properly regarded as an interval scale representation of the original points. Table 1 presents lower and upper bounds on the estimated value of CM. Note that $\mathrm{CM}$ is estimated to be above .995 in all cases. ${ }^{3}$ Given these high values for $\mathrm{CM}$, the projection values can be taken as representing interval scale measurement.

Figure 2 plots the projection values as a function of decibels of sound pressure for each subject and for the group data. Since the projection values are unique only up to addition and multiplication by a constant, they have been normalized to have the same range (1.0) and mean value (.3). Note that the points are positively accelerated in these coordinates and that the degree of acceleration varies from individual to individual, with the degree of acceleration for the group data falling between the two extremes.

It is easy to show that the positive acceleration in Figure 2 is inconsistent with Fechner's law. Since the projection value in Figure 2 constitute interval scale measurement, they should be linearly related to loudness ( $P=a L+b$, where $a$ and $b$ are constants). Fechner's law states that loudness is a logarithmic function of intensity, that is, that loudness is linear with decibels $[\mathrm{L}=\mathrm{c}(\mathrm{dB})+\mathrm{k}$, where $\mathrm{c}$ and $\mathrm{k}$ are constants]. By substitution, then, the projection values should be linear with decibels. Figure 2 shows that this is clearly not the case. Consequently, Fechner's law cannot account for the paired comparison data.

However, if loudness is described by Stevens' law, then $L=k I^{n}$. Since $P=a L+b$, by substitution we obtain $P=a^{\prime} I^{n}+b$, where $a^{\prime}=a k$. Equivalently, $(P-b)=a^{\prime} I^{n}$. Taking logs of both sides of the equation, Stevens' law describes the present data if there

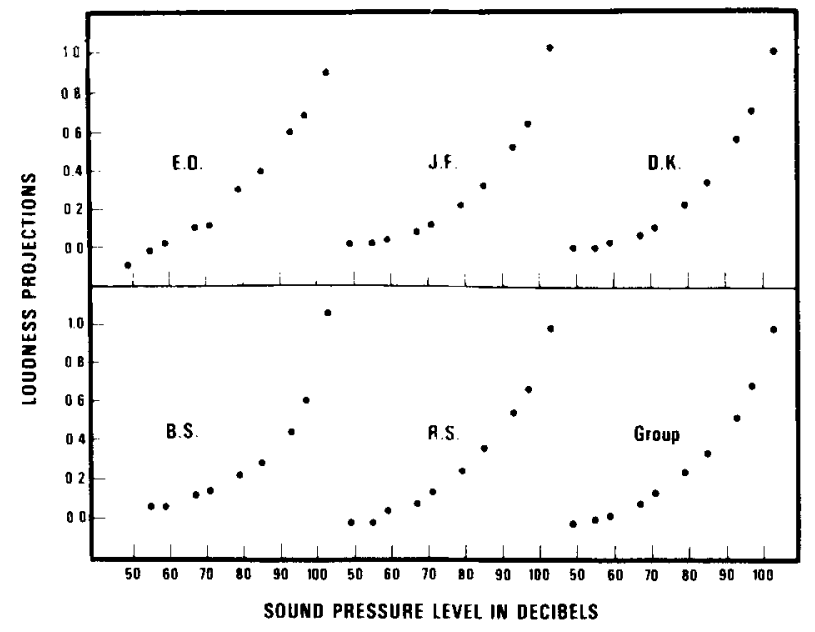

Figure 2. Loudness projections as a function of decibels of sound pressure for five subjects considered individually and as a group.

is a b such that $\log (P-b)$ is linear with stimulus intensity in decibels. For each of the five subjects and for the group data, the value of $b$ that maximized the square correlation coefficient $\left(r^{2}\right)$ between log $(\mathrm{P}-\mathrm{b})$ and $\mathrm{dB}$ was determined. The projection values adjusted by this value of $b$ are called the adjusted loudness projections.

Figure 3 plots the adjusted loudness projections (logarithmic spacing) against decibels of sound pressure. Since the definition of unit is arbitrary in these coordinates, the adjusted loudness projections for each subject have been multiplied by a constant so that the geometric mean value is 8 . The straight lines drawn through the points were determined by the method of least squares and provide reasonably good fits to the data. The exponents of the power functions for the subjects (see Table 1) range from .11 to .28 , with the exponent for the group being .21 .

Intersubject differences. If all subjects had the same loudness scale, then the projection values shown in Figure 2 would be identical except for whatever deviations could be attributed to noise in the measurement process. The index of coordinate metric determinacy can be used as an indicator of the accuracy with which individual loudness scales are assessed. Since CM is above .995 in all cases, the expectation is that the squared Pearson correlation coefficient between the underlying representation of loudness for a subject and the projection values from the nonmetric program exceeds .995 . Hence, if two subjects shared the same loudness scale, we would expect an $r^{2}$ of at least .990 between their projection values. ${ }^{4}$ This expectation is violated in the present case for two of the subjects. The $r^{2}$ values for Subject B.S. vs. the other four subjects range from .889 to .979 . Also, the $\mathrm{r}^{2}$ values for Subject E.D. vs. the other four range from .889 to .980 . (The $\mathrm{r}^{2}$ for B.S. vs. 


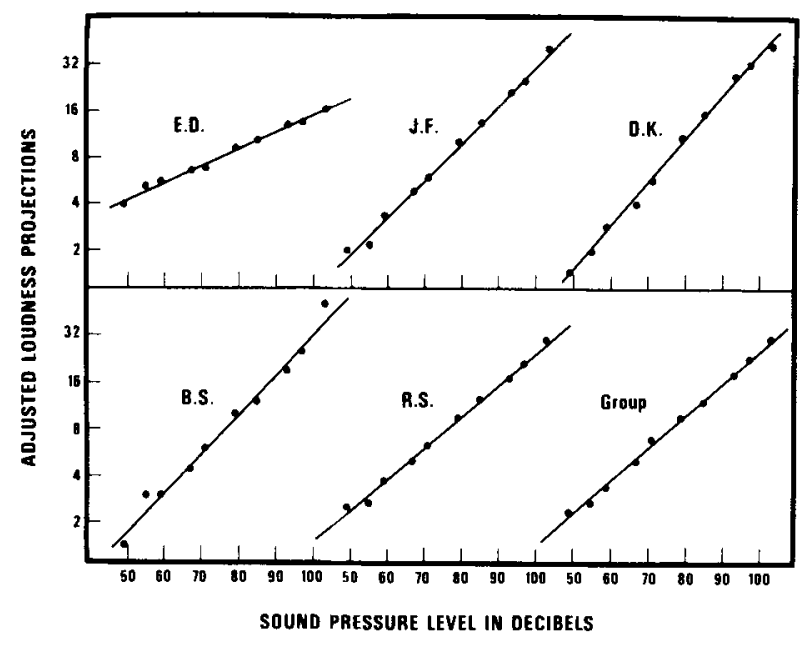

Figure 3. Adjusted loudness projections (see text) as a function of decibels of sound pressure for five subjects considered individually and as a group. Note that the ordinate is spaced logarithmically. A straight line in these coordinates indicates that a power function describes the growth of loudness as a function of sound intensity.

E.D. is .889.) There is no evidence based on $r^{2}$ values that the other three subjects differ significantly from one another since the $\mathrm{r}^{2}$ values for any pairing among the three exceed .994.

Intrasubject variability. For each subject, every eight sessions constituted a replication of the basic experiment; that is, over the course of eight sessions, each subject judged each of the 540 comparisons exactly once. In order to look for possible changes over time, projection values were obtained from the nonmetric program for each of the eight replications. Values of $G$ for each of these replications are given in Table 2. As expected, $G$ is higher since each solution is based on only one instead of eight judgments per stimulus pair. The highest $G$ value for a subject can be used as a conservative estimate of the precision with which the underlying loudness scale values have been recovered. As can be seen from Table 2, these conservative estimates would place the value of CM at or above .995 for all subjects.

Figure 4 plots the scale values obtained from the nonmetric analysis as a function of decibels of sound pressure. Again, the projection values have been

Table 2

Values of $\mathrm{G}$ and Estimate of $\mathrm{CM}$

\begin{tabular}{lccccccccc}
\hline & \multicolumn{7}{c}{ Replication } \\
\cline { 2 - 8 } $\begin{array}{c}\text { Sub- } \\
\text { ject }\end{array}$ & 1 & 2 & 3 & 4 & 5 & 6 & 7 & 8 & CM \\
\hline E.D. & .131 & .131 & .107 & .107 & .113 & .124 & .117 & .126 & .995 \\
J.F. & .106 & .104 & .113 & .120 & .109 & .104 & .120 & .087 & .995 \\
D.K. & .102 & .117 & .098 & .120 & .131 & .113 & .122 & .124 & .995 \\
B.S. & .044 & .039 & .043 & .044 & .030 & .030 & .033 & .056 & .998 \\
R.S. & .074 & .067 & .056 & .057 & .067 & .044 & .057 & .059 & .997 \\
\hline
\end{tabular}

normalized to have the same range $(1.0)$ and the same average value $(.3)$. The values across replications overlapped to such an extent that it was not possible to identify individual replications on this graph. ${ }^{5}$ Figure 4 shows that Subjects B.S. and R.S. have the lowest degree of variance across replications, while Subjects D.K., J.F., and E.D. are more variable. This visual impression is confirmed by examining the mean $\mathrm{r}^{2}$ across all pairs of replications $\left(\mathrm{r}^{2}=.996\right.$, $.993, .988, .987$, and .986 for Subjects R.S., B.S., D.K., J.F., and E.D., respectively) ${ }^{6}$ Note that, with the exception of Subject R.S., these mean $r^{2} s$ are less than we would expect under the hypothesis that the subject's loudness values are unchanging from replication to replication. Since the value of CM is the expected squared Pearson correlation coefficient between the underlying loudness scale and the projection values obtained on any single replication, the average $r^{2}$ should be about .990 for Subjects E.D., J.F., and D.K., and .992 and .996 for Subjects R.S. and B.S., respectively (see Footnote 4). Since it is lower for four of the five subjects, there is clearly some variability, however small, in the underlying loudness values.

If the variability in the underlying loudness scale is random in nature, then the $r^{2}$ values between projection values obtained for consecutive replications should be the same as the $r^{2}$ values between projection values obtained from replications more widely separated in time; that is, the average $\mathrm{r}^{2}$ for replications 1 and 2,2 and 3,3 and $4, \ldots .7$ and 8 should be the same as the average for replications 1 and 4 , 2 and $5, \ldots .5$ and 8 . If, on the other hand, the underlying loudness scale was changing in a systematic way, the $r^{2}$ for consecutive replications should be greater than the $r^{2}$ for replications more widely separated in time. Figure 5 plots the mean $r^{2}$ between replications as a function of the number of intervening replications. Hence, the number 0 on the abscissa represents the consecutive replications ( 1 and 2, 2 and 3,3 and 4, 4 and 5, 5 and 6,6 and 7,7 and 8), the number 1 represents the replications separated by exactly one replication ( 1 and 3,2 and 4,3 and 5, 4 and 6,5 and 7,6 and 8 ), while the number 3 represents the replications separated by exactly three replications ( 1 and 5, 2 and 6, 3 and 7, 4 and 8). (The functions are not plotted for replications separated by more than three replications since the number of $\mathrm{r}^{2}$ values entering into the average becomes too small.) Figure 5 shows that, for three of the subjects, there is no indication of any trend; however, for two subjects (E.D. and J.F.), $r^{2}$ values may be decreasing somewhat over time, although the trend is not strong. It is interesting to note that the two subjects who appear to be the least stable across replications also have the greatest number of intransitivities (see Table 1). 


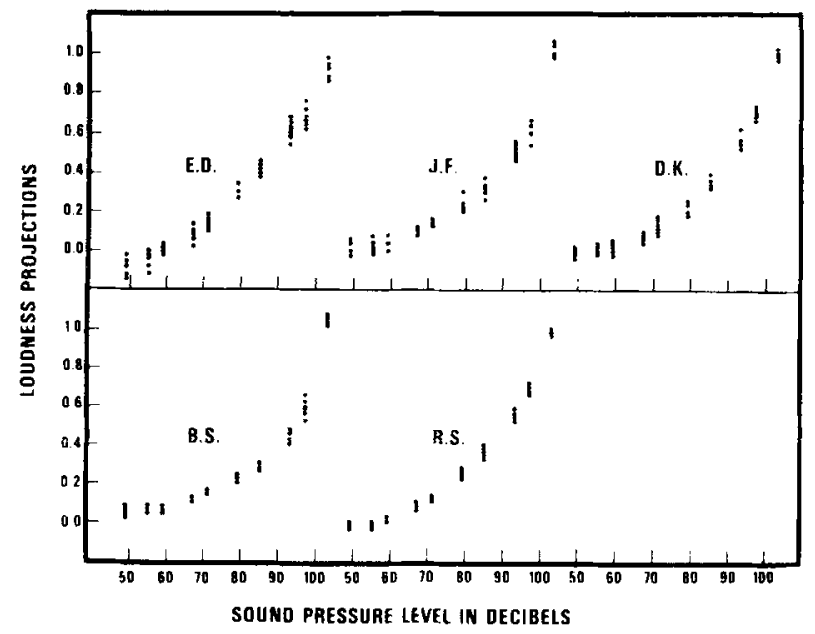

Figure 4. Loudness projections as a function of decibels of sound pressure for five subjects. For each subject, the projection values from each of eight replications are superimposed.

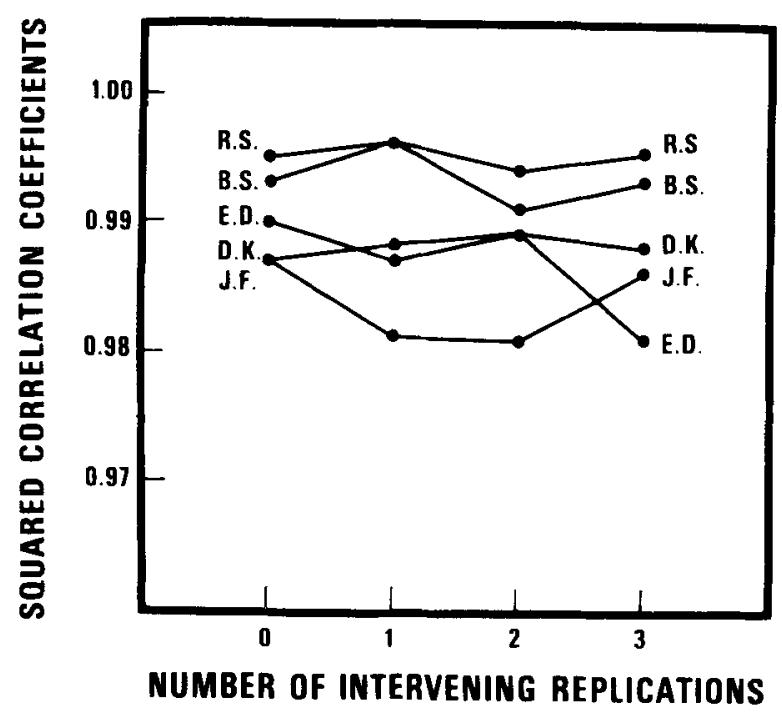

Figure 5. Averaged squared correlation coefficients between replications for each of five subjects as a function of the number of intervening replications.

If the projection values from each replication are based on the same loudness values, then a single exponent should be sufficient to describe the growth of loudness for all replications. To assess the extent to which this was true for the present data, an exponent was determined that would minimize the variance from a straight line in $\log -\log$ coordinates. The results are shown in Figure 6. It can be seen from Figure 6 that a single exponent provides a good fit to the data for Subjects E.D., B.S., and R.S. A power function in these coordinates with a single exponent for all eight replications accounts for $98.9 \%, 98.7 \%$, and $99.2 \%$ of the variance for Subjects E.D., B.S., and R.S., respectively. By way of contrast, fitting a separate exponent to each of the eight replications for these three subjects can only account for an additional $.1 \%$ of the variance. Hence, for these three subjects, fitting a single exponent to the data from all eight replications accounts for almost the same proportion of variance as fitting a separate exponent to the data from each of the eight replications. The values of the best fitting exponents for E.D., B.S., and R.S. are $.12, .28$, and .21 . Note that these values correspond quite closely to those that were obtained when the loudness interval comparisons were collapsed over the eight sessions (see Table 1).

Figure 6 also shows that although a power function describes the growth of loudness for J.F. and D.K., these subjects are more variable across replications. Fitting a single exponent to all eight replications (see Figure 6) accounted for $98.3 \%$ and $97.9 \%$ of the variance, while fitting separate exponents to each replication accounted for $99.2 \%$ and $98.7 \%$ of the variance for Subjects J.F. and D.K. Hence, there was an increase of about $1 \%$ in the percentage of variance accounted for when eight separate exponents, instead of a single exponent for all eight replications, were fitted to the data. The values of the best fitting exponents for J.F. and D.K. in Figure 6 are .21 and .20 , respectively. In contrast with the results from Subjects E.D., B.S., and R.S., these values do not correspond as closely to those determined when the data were collapsed across the eight replications (see Table 1). Since the variance across replications in these coordinates is greatest for these two subjects, it is perhaps not surprising that the two methods of estimating the loudness exponent show the greatest degree of disagreement in this case.

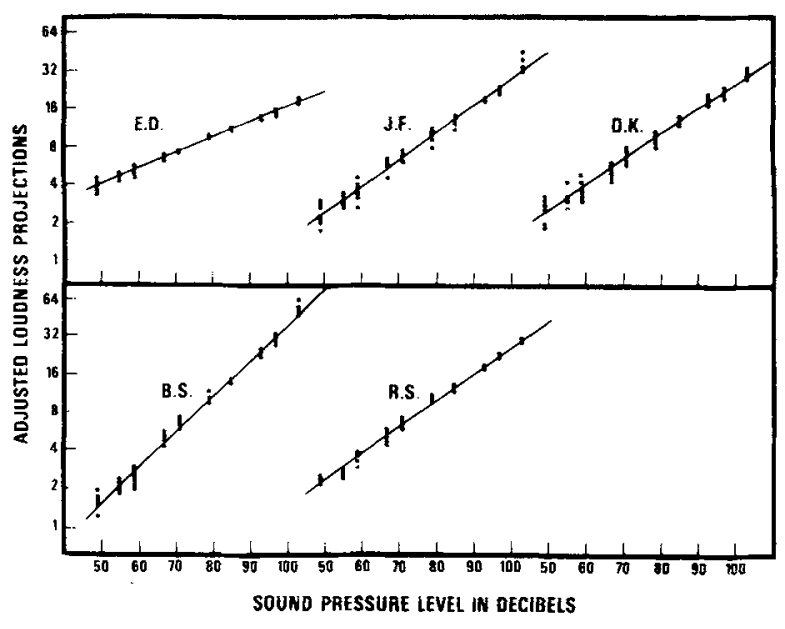

Figure 6. Adjusted loudness projections (see text) as a function of decibels of sound pressure. For each subject, the projection values from each of eight replications are superimposed. Note that the ordinate is spaced logarithmically. A straight line in these coordinates indicates that a power function describes the growth of loudness as a function of intensity. 


\section{DISCUSSION}

\section{Scale Validity}

In any psychophysical scaling procedure, there is always some question of whether the subject is behaving in accordance with the instructions. For example, in magnitude estimation, the subject is in structed to make his numerical assignments proportional to the loudnesses of the tone. The extent to which the subject can or does comply with this request determines the validity of the loudness scale derived from his numerical estimates. Consequently, it seems sensible to construct scales of loudness from sensory judgments whose formal properties are testable (e.g., Anderson, 1977; Krantz et al., 1971). In the present experimental paradigm, transitivity and monotonicity test whether or not the loudness interval comparisons are consistent with a model in which a pair of tones $(x, y)$ is judged to have a larger loudness interval than another pair $(w, z)$ if and only if $|L(y)-L(x)|>|L(z)-L(w)|$. Since, for all subjects, violations of transitivity and monotonicity were quite low, it is reasonable to assume that the subjects were behaving in accordance with the instructions, that is, that their loudness interval comparisons were actually based on the loudness differences between tones. Consequently, the projection values obtained from the nonmetric scaling program constitute interval scale measurement of loudness. Figure 3 shows that these interval scales of loudness, after suitable adjustment by an additive constant, are power functions of sound intensity.

\section{Intra- and Intersubject Differences}

An examination of Figure 4 indicates that the loudness scale values for a subject are relatively stable across replications. However, Figures 2 and 4 suggest that there are strong individual differences. This can be seen most clearly if we plot the loudest scale values from each of the eight replications for a pair of subjects on a single graph. This is done in Figure 7 for Subjects B.S. and R.S. (Recall that the loudness values from each replication have been adjusted to have the same range and mean value, i.e., to be as comparable as possible.) Figure 7 shows that the growth of loudness with intensity is quite different for B.S. and R.S., and that this difference cannot be attributed to imprecision or error in the measurement process, since for 6 of the 10 stimuli there is no overlap in the distributions of loudness values. A two-way analysis of variance with subjects and replications as factors (using the interaction term as the error term) confirms that there is a significant subject effect $[F(4,28)$ $=4.40, \mathrm{p}<.01]$ and no effect due to replication $[\mathrm{F}(7,28)=.29, \mathrm{p}>.9]$.

It is important to note that these differences among individuals cannot be easily attributed to differences

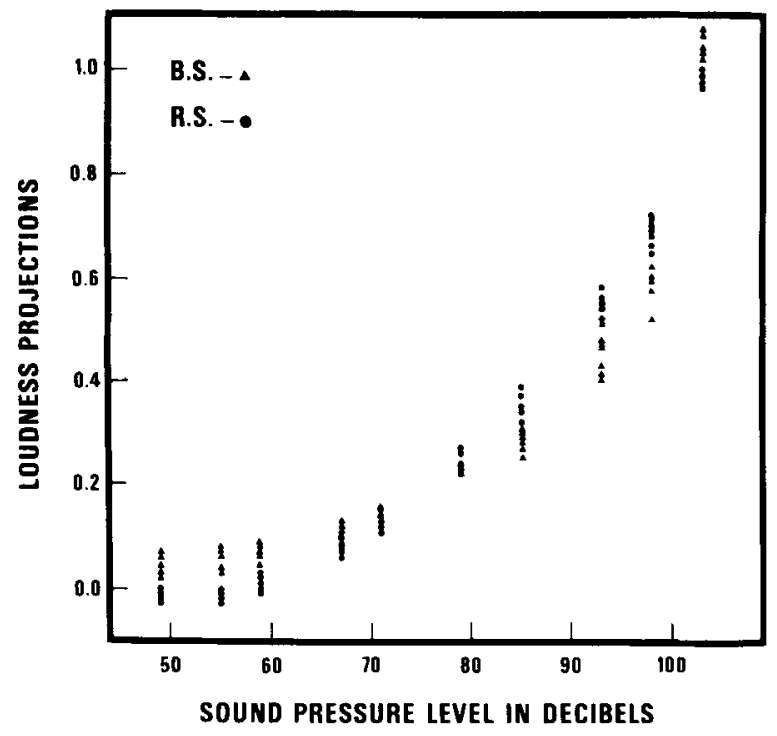

Figure 7. Loudness projections for Subjects B.S. and R.S. superimposed on the same graph. The loudness projections from each of the eight replications for each subject are shown.

in the way subjects employ numbers or numerical concepts. First, the explicit use of numbers was avoided in the present experiment by asking subjects to directly compare loudness intervals. Second, while it is possible to hypothesize that subjects were, in fact, making numerical judgments of the two tones in a pair, determining their numerical difference, and then comparing this numerical difference with its counterpart for the second pair of tones, introspective evidence obtained from all five subjects, and the speed with which the judgments were made in some instances, make such an explanation unlikely. Therefore, there appear to be real intersubject differences in the perception of loudness intervals.

These intersubject differences suggest that the large range of loudness exponents obtained across subjects when direct estimation procedures are employed (e.g., Stevens \& Guirao, 1964) may not be attributable solely to the way in which subjects employ numbers or numerical concepts, but may, to some extent, reflect differences in the way in which intensity information is coded or processed in different individuals. It would be interesting to determine the extent to which such individual differences show up in other scaling paradigms that are also free from numerical biases. For example, Levelt, Riemersma, and Bunt (1972) constructed loudness scales from binaural additivity judgments using a conjoint measurement approach. In their experiment, subjects listened to dichotically presented $1,000-\mathrm{Hz}$ tones whose left- and right-ear sound pressures differed. On a single trial, a subject was presented with two such tones in sequence and asked to indicate which tone appeared louder. Hence, numerical judgments were avoided. Since their results were consis- 
tent with binaural loudness additivity, they constructed scales of loudness for the left and right ears of two individuals. For one subject, the exponents for the left and right ears were .205 and .235 , respectively, while for the other subject, the exponents for the left and right ear were .220 and .305 . Hence, there is some indication that there are differences across individuals and that the left and right ears of a single individual might differ. Unfortunately, the degree of metric determinacy for these data is not known, and it is not possible to say whether these obtained differences in loudness functions are significant. However, if further studies of this sort indicate individual differences in the value of the loudness exponent, they will provide further evidence that the growth of loudness with intensity differs from individual to individual.

In theory, individual differences in the coding of sound intensity could be introduced at any level of sensory processing, starting with the transduction of mechanical energy into neural impulses in the inner ear. The present results can in no way pinpoint the locus or loci of these individual differences. However, since differences could occur anywhere from the initial neural encoding in the inner ear to the point at which the two tone intensities are compared, it is conceivable that these individual differences are more "cognitive" than sensory in origin. Only a series of studies designed to assess the sensory code at various stages of processing will be able to determine the sources of these individual differences. (See Luce, 1972, for a discussion of the consequences of such individual differences for measurement theory.)

\section{Comparisons with Other Studies}

Figure 8 plots the loudness projections from Schneider et al. (1974) and from the group data of the present study as a function of decibels of sound pressure. Tonal intensities were $1 \mathrm{~dB}$ higher in Schneider et al. (1974). In all other respects, the stimuli and procedures were comparable. Again, loudness projections have been adjusted to have a mean of .3 and a range of 1.0. Note that in both studies the growth of loudness as a function of sound intensity is quite similar. However, the function from the present study shows a slightly greater degree of acceleration at the lower intensity values. This greater acceleration is responsible for the difference in exponent values found in the two studies-.15 for Schneider et al. (1974) and .21 for the group data in the present study. The large intersubject differences in the growth of loudness found in the present study (see Figures 2 and 7) might easily produce a difference of this order. Hence, the two studies are in reasonably good agreement.

These exponents for direct comparisons of loudness intervals are also in good agreement with those

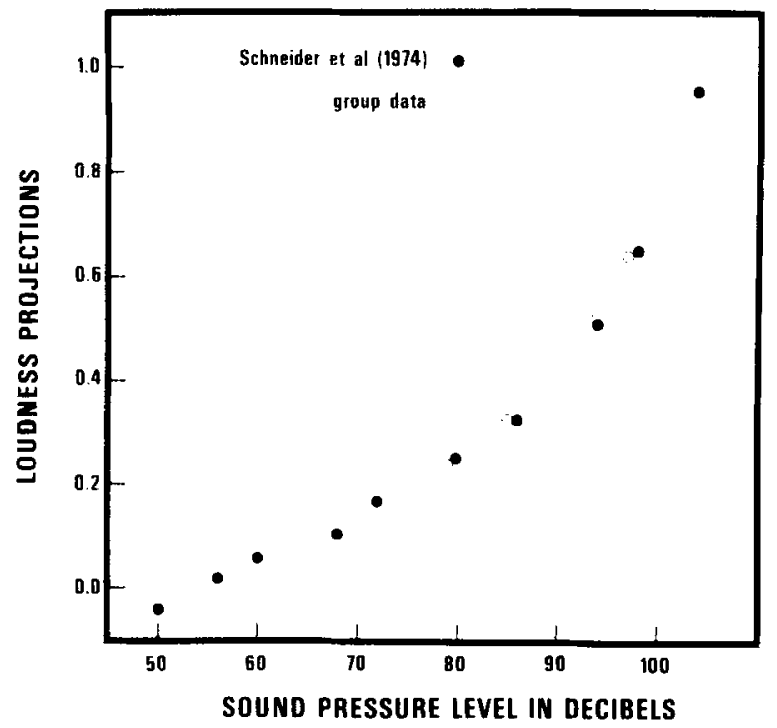

Figure 8. Loudness projections from Schneider et al. (1974) and for the group of five subjects as a function of decibels of sound pressure.

found when direct estimates of loudness intervals are obtained. Schneider et al. (1978) showed that a power function with an exponent of .145 described the loudness scales obtained from a nonmetric analysis of direct judgments (both magnitude and category) of loudness differences and loudness similarities for pairs of $1,200-\mathrm{Hz}$ tones. It should be noted that these exponents, which are based on judgments of loudness intervals, are somewhat lower than the exponent of .27 that has been suggested for monaural pure tones (Reynolds \& Stevens, 1960) but agree fairly well with Garner's $(1954,1959)$ lambda scale of loudness and with a functional measurement analysis of bisection judgments of loudness (Carterette \& Anderson, 1979). Several explanations of this discrepancy have been suggested. S. S. Stevens (1971) has argued that subjects are unable to judge equal intervals at different locations on a prothetic continuum. When they attempt to do so, a constant difference seems larger toward the lower, as compared with the higher, end of the continuum. This asymmetrical distortion, Stevens argues, lowers the estimate of the exponent. Note, however, that this argument assumes the validity of the exponent based on direct estimation techniques.

Marks (1974) has argued that the sensory representation for sensory differences is simply different from that for sensory magnitude. Hence, scales obtained from direct estimation procedures measure a sensory phenomenon different from those obtained from scaling of sensory intervals.

Finally, as mentioned in the introduction, many investigators have argued that the estimate of the exponent obtained from judgments of sensory magnitude is biased by the way in which subjects use 
numbers. Hence, it is possible that the apparent disagreement in exponents is illusory, for, once it is assumed that numerical judgments may not be directly proportional to sensation, the most that can be said is that the value of the exponent obtained in magnitude estimation and cross-modality matching experiments is unique only up to multiplication by a constant (e.g., Krantz, 1972; Schneider et al., 1974). Hence, depending on the form numerical biases take, the direct estimation techniques may be in agreement with the nonmetric interval scaling techniques.

The present results are not able to resolve these competing theories. However, if numerical biases are present, it seems sensible to base loudness scales on judgments that are free of these biases. In this way, individual differences in the growth of loudness can be assessed with some confidence that these differences are not an artifact of the way in which subjects employ numbers or numerical concepts.

\section{REFERENCES}

ANDERson, N. H. Cross-task validation of functional measurement using judgments of total magnitude. Journal of Experimental Psychology, 1974, 102, 226-233.

Anderson, N. H. Note on functional measurement and data analysis. Perception \& Psychophysics, 1977, 21, 201-215.

Carterette, E. C., \& Anderson, N. H. Bisection of loudness. Perception \& Psychophysics, 1979, 26, 265-280.

Curtis, D. W., Attineave, F., \& Harrington, T. L. A test of a two-stage model of magnitude judgment. Perception \& Psychophysics, 1968, 3, 25-31.

Cuntis, D. W., \& RulE, S. J. Magnitude judgments of brightness and brightness difference as a function of background reflectance. Journal of Experimental Psychology, 1972, 95, 215-222.

Fagot, R. F., Stewart, M. R., \& Kleinknecht, R. E. Representations for biased numerical judgments. Perception \& Psychophysics, 1975, 17, 309-319.

Garner, W. R. A technique and a scale for loudness measurement. Journal of the Acoustical Society of America, 1954, 26, 73-88.

Garner, W. R. On the lambda loudness function, masking, and the loudness of multicomponent tones. Journal of the Acoustical Society of America, 1959, 31, 602-607.

Hays, W. L. Statistics for the social sciences. New York: Holt, Rinehart \& Winston, 1973.

HUBERT, L. Seriation using asymmetric proximity measures. British Journal of Mathematical and Statistical Psychology, $1976,29,32-52$.

Kendal, M. G., \& Babington Smith, B. On the method of paired comparisons. Biometrika, 1939, 31, 324-345.

KRANTZ, D. H. A theory of magnitude estimation and crossmodality matching. Journal of Mathematical Psychology, 1972, 9, 168-199.

Krantz, D. H., Luce, R. D., Suppes, P., \& Tversky, A. Foundations of measurement (Vol. 1): Additive and polynomial representation. New York: Academic Press, 1971.

Levelt, W. J. M., Riemersma, J, B., \& Bunt, A. A. Binaural additivity of loudness. British Journal of Mathematical and Statistical Psychology, 1972, 25, 51-68.

LucE, R. D. What sort of measurement is psychophysical measurement? American Psychologist, 1972, 27, 96-106.

Marks, L. E. On scales of sensation: Prolegomena to any future psychophysics that will be able to come forth as science. Perception \& Psychophysics, 1974, 16, 358-376.
Marks, L. E., \& Stevens, J. C. Individual brightness functions. Perception \& Psychophysics, 1966, 1, 17-24.

Morrison, D. Multivariate statistical methods. New York: McGraw-Hill, 1976.

Reynolds, G. S., \& Stevens, S. S. Binaural summation of loudness. Journal of the Acoustical Society of America, 1960, 32, 1337-1344.

Rule, S. J., Curtis, D. W., \& Markley, R. P. Input and output transformations from magnitude estimation. Journal of Experimental Psychology, 1970, 86, 343-349.

Rule, S. J., LAYE, R. C., \& Curtis, D. W. Magnitude judgments and difference judgments of lightness and darkness: A twostage analysis. Journal of Experimental Psychology, 1974, 103, $1108-1114$.

Rule, S., \& Markely, R. P. Subject differences in cross-modality matching. Perception \& Psychophysics, 1971, 9, 115-117.

Schneider, B. A technique for the nonmetric analysis of paired comparisons of psychological intervals. Psychometrika, 1980, 45, 357-372.

Schneider, B., Parker, S., \& Stein, D. The measurement of loudness using direct comparisons of sensory intervals. Journal of Mathematical Psychology, 1974, 11, 259-273.

Schneider, B., Parker, S., Valenti, M., Farrell, G., \& KANow, G. Response bias in category and magnitude estimation of difference and similarity for loudness and pitch. Journal of Experimental Psychology: Human Perception and Performance, $1978,4,483-496$.

Shepard, R. N. Metric structures in ordinal data. Journal of Mathematical Psychology, 1966, 3, 287-315.

Slater, P. Inconsistencies in a schedule of paired comparisons. Biometrika, 1961, 48, 303-312.

Stevens, J. C., \& Guirao, M. Individual loudness functions. Journal of the Acoustical Society of America, 1964, 36, 22102213.

Stevens, S. S. The measurement of loudness. Journal of the Acoustical Society of America, 1955, 27, 815-829.

Stevens, S. S. The direct estimation of sensory magnitudeloudness. American Journal of Psychology, 1956, 69, 1-15.

Stevens, S. S. Issues in psychophysical measurement. Psychological Review, 1971, 78, 426-450.

WALSH, J. K., \& Brow MaN, C. P. Intraindividual consistency on a cross-modality matching task. Perception \& Psychophysics, $1978,23,210-214$.

Wanschura, R. G., \& Dawson, W. E. Regression effect and individual power functions over sessions. Journal of Experimental Psychology, 1974, 102, 806-812.

\section{NOTES}

1. Schneider et al. (1974) actually reported an exponent of .13. The .15 value cited here is based on a reanalysis of their data using a nonmetric scaling program (Schneider, 1980) specifically designed for the analysis of paired comparisons of sensory intervals.

2. Finding the minimum number of violations of Equation 5 is the same as finding the minimum number of reversals of the comparisons of loudness intervals that would be necessary to satisfy transitivity. Slater (1961) refers to the minimum number of reversals necessary to achieve transitivity as the minimum number of inconsistencies in a set of paired comparisons. Thus, we are not referring to the number of circular triads, which is another common way of evaluating transitivity (Kendall \& Babington Smith, 1939). In determining the minimal number of inconsistencies, the set of permissible rank orders was restricted to those orders that did not violate the nesting condition. For example, in all orders, the 93-103-dB interval had to be ranked higher than the 97-103-dB interval, and so on. As Hubert (1976) points out, determining the set of rank orders that minimizes inconsistencies is, in general, not a trivial task. However, if the number of incon- 
sistencies is small, as it was in this case, it can be done by hand using any of a number of different methods. One disadvantage of this technique for determining the number of violations of transitivity is that the distribution of the number of inconsistencies is not known for large $n$ (Slater, 1961).

3. This lower estimate is conservative. In its computation, all of the ties are assumed to be disagreements between predicted and obtained comparisons (Schneider, 1980). A more likely lower bound on the estimate of coordinate metric determinacy would be on the order of .997 .

4. If two variables, $X_{1}$ and $X_{2}$, are both correlated with $X_{3}$, then the partial correlation coefficient $Q_{12}$, measures the degree of relationship between $X_{1}$ and $X_{2}$ that cannot be attributed to the mutual correlation with $X_{3}$. Hence, if two sets of projection values are both correlated to the same extent with the "true" loudness values, and if these "true" loudness values are the same for both sets of projection values, then the partial correlation coefficient between the two sets of projection values should be zero. In order for the partial correlation coefficient to be zero under these circumstances (see Morrison, 1976), it is required that $\varrho_{12}=\left(\varrho_{13}\right) \times$ $\left(Q_{23}\right)$. In our case, $Q_{13}$ is the hypothesized correlation between the first set of projection values and the true loudness values, while $Q_{23}$ is the correlation between the second set and the true loudness values. Both are estimated as $\mathrm{CM}^{1 / 2}$. Hence, the estimated value of $Q_{12}$ (the expected correlation coefficient between two sets of projection values) should be $C M$ and $Q_{12}^{2}$ should be $\mathrm{CM}^{2}$. Unfortunately, confidence intervals on this estimate are defined only for bivariate normal distributions. Since the projection values obtained from a nonmetric program are not independent, standard statistical tests do not apply. Hence, the expected value can only be used as a guideline.

5. When these projection values are averaged over replications, the average values are nearly identical to the projection values shown in Figure 2 for each subject. A regression of the Figure 2 values against the average values in Figure 4 yield $r^{2}$ values in excess of .9985 for each subject. Hence, these two ways of esti- mating characteristic scale values for a subject yield essentially the same result.

6. Average squared correlation coefficients were determined using Fisher's transformation (see Hays, 1973). Specifically, correlation coefficients were changed to $Z$ scores by means of Fisher's transformation, and the mean $Z$ score was computed and transformed back into a correlation coefficient. This correlation coefficient was then squared to yield the mean $r^{2}$ value. Fisher's method is used in this paper whenever correlation coefficients are averaged.

7. The projection values from a particular replication are unique up to addition and multiplication by a constant. Hence, in determining the best fitting exponent for the data, the projection values from each replication $\left(\mathrm{P}_{\mathrm{i}}\right)$ can be adjusted by additive and multiplicative constants in order to produce the best fit. This was accomplished in the following way. First, for each replication, an additive constant, $b_{i}$, was determined such that the regression of $\log \left(\mathrm{P}_{\mathrm{i}}-\mathrm{b}_{\mathrm{i}}\right)$ on $\mathrm{dB}$ produced a specified value of the slope $(\mathrm{n})$. The value of $n$ in this first step was held constant across replications. In the second step of the minimization process, the $\left(\mathrm{P}_{\mathrm{i}}-\mathrm{b}_{\mathrm{i}}\right)$ for each replication was adjusted by a multiplicative constant, $k_{i}$, such that $\Sigma \log k_{1}\left(P_{1}-b_{1}\right)=\Sigma \log k_{2}\left(P_{2}-b_{2}\right)=$ $\ldots=\Sigma \log k_{8}\left(P_{s}-b_{8}\right)=9.0$. It can be shown that the regression of the projection values adjusted in this fashion on $\mathrm{dB}$ will produce the same value of the slope and intercept for each replication. Hence, these values of $b$ and $k$ will minimize $r^{2}$ for a particular value of $n$ when all of the $\log k_{i}\left(P_{i}-b_{i}\right)$ projection values are regressed on $\mathrm{dB}$. In the third and final step, the value of $n$ was changed and the first two steps repeated until a value of $n$ was found such that $r^{2}$ was minimized. The adjusted projection values that produced the value of $n$ that minimized $r^{2}$ are shown in Figure 6.

(Received for publication June 2, 1980; revision accepted August 25, 1980.) 